\title{
Erratum
}

Med Klin Intensivmed Notfmed 2021 · 116:360 https://doi.org/10.1007/s00063-021-00819-y Online publiziert: 20. April 2021

(c) Springer Medizin Verlag GmbH, ein Teil von Springer Nature 2021
J. Prinz' $\cdot$ B. Böll' $\cdot$ M. von Bergwelt-Baildon ${ }^{2} \cdot$ V. Burst ${ }^{3} \cdot$ J. U. Becker ${ }^{4} \cdot$ D. CarvalhoFiel $^{5} \cdot$ A. Shimabukuro-Vornhagen ${ }^{1} \cdot$ M. Kochanek ${ }^{1}$

'Klinik I für Innere Medizin, Universitätsklinikum Köln, Köln, Deutschland

${ }^{2}$ Medizinische Klinik und Poliklinik III, Klinikum der Universität München, München, Deutschland

${ }^{3}$ Klinik II für Innere Medizin, Universitätsklinikum Köln, Köln, Deutschland

${ }^{4}$ Institut für Pathologie, Universitätsklinikum Köln, Köln, Deutschland

${ }^{5}$ Serviço de Nefrologia, Hospital do Espírito Santo, Évora, Portugal

\section{Erratum zu: Intoxikation nach Frostschutzmittelaufnahme}

\section{Der Fall eines Patienten nach wiederholter Ethylenglykolintoxikation}

\section{Erratum zu:}

Med Klin Intensivmed Notfmed 2018

https://doi.org/10.1007/s00063-018-

0439-5

In diesem Beitrag wurde der Name des Autors M. von Bergwelt-Baildon falsch wiedergegeben. Bitte beachten Sie die korrigierte Schreibweise. Der ursprün gliche Artikel wurde korrigiert

\section{Korrespondenzadresse}

\section{Dr. J. Prinz}

Klinik I für Innere Medizin, Universitätsklinikum Köln

Kerpener Str. 62, 50937 Köln, Deutschland johanna.prinz@uk-koeln.de
Die Online-Version des Originalartikels ist unter https://doi.org/10.1007/s00063-018-0439-5 zu finden. 